\title{
The INES system
}

\section{Evaluation of IUE NEWSIPS high resolution spectra}

\author{
R. González-Riestra ${ }^{1, \star}$, A. Cassatella ${ }^{2,3, \star}$, E. Solano ${ }^{1, \star}$, A. Altamore ${ }^{3}$, and W. Wamsteker ${ }^{4, \star \star}$ \\ 1 Laboratorio de Astrofísica Espacial y Física Fundamental, VILSPA, P.O. Box 50727, E-28080 Madrid, Spain \\ 2 CNR, Istituto di Astrofisica Spaziale, Via del Fosso del Cavaliere, I-00133 Roma, Italy \\ 3 Dipartimento di Fisica E. Amaldi, Universitá Roma Tre, Via della Vasca Navale 84, I-00146 Roma, Italy \\ 4 ESA-IUE Observatory, VILSPA, P.O. Box 50727, E-28080 Madrid, Spain
}

Received July 23; accepted October 22, 1999

\begin{abstract}
This paper discusses the overall quality of IUE high resolution data processed with the NEWSIPS software in terms of flux and wavelength accuracy. It also describes the processing of NEWSIPS high resolution spectra within the framework of the ESA "IUE Newly Extracted Spectra" (INES) System. This system provides the IUE high resolution data in two formats: the high resolution "concatenated" spectra, in which the spectral orders are connected, eliminating the overlap regions through a procedure designed to optimize the signal-to-noise ratio at the edges of the orders, and the "rebinned" spectra, i.e. the high resolution concatenated spectra resampled into the low resolution wavelength domain. Our study reveals the existence of a significant discrepancy in the wavelength scales of short and long wavelength NEWSIPS high resolution spectra. The INES processing applies a correction of $+17.7 \mathrm{~km} \mathrm{~s}^{-1}$ to the wavelength scale of the high resolution SWP spectra in order to provide an internally consistent velocity scale. Similarly, suitable corrections have been applied to long wavelength small aperture spectra. The new wavelength scale is, within the errors, in good agreement with the optical velocity scale.
\end{abstract}

Key words: methods: data analysis; techniques: image processing; ultraviolet: general

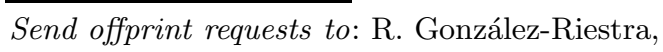

e-mail: ch@laeff.esa.es

* Previously: ESA-IUE Observatory.

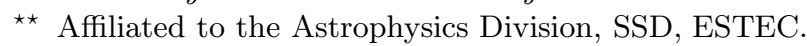

\section{Introduction}

The IUE NEWSIPS processing system was developed with the aim of creating a "Final Archive" of $I U E$ data to be made available to the astronomical community as a legacy after the end of the project. This archive would include all the $I U E$ spectra re-processed with improved algorithms and up-to-date calibrations. The NEWSIPS processing system is fully described by Nichols \& Linsky (1996) and Nichols (1998). Technical details are given in the NEWSIPS Manual (Garhart et al. 1997). The introduction of new techniques to perform the geometric and photometric corrections led to a substantial improvement in the signal-to-noise ratio of the final spectra. Further improvements in the quality of the high resolution data arise from the new method to determine the image background and from the improved ripple correction and absolute calibration (Cassatella et al. 1999, hereinafter Paper II). The background subtraction has always been one of the more critical issues in the processing of IUE high resolution spectra, particularly at the shortest wavelengths, where orders crowd and an accurate estimate of the background is essential for a correct flux extraction. This problem has been overcome in NEWSIPS through the derivation of a bi-dimensional background (see Smith 1999 for a description of the method).

The goal of the INES processing system was to correct the deficiencies found during the scientific evaluation of the data processed with NEWSIPS for the IUE Final Archive, and to provide the output data to the users in a simple way requiring a minimum knowledge of the operational and instrumental characteristics of IUE (Wamsteker et al. 1999). The modifications introduced in the processing of low resolution data have been described by Rodríguez-Pascual et al. (1999, Paper I). As for high resolution data, the INES system provides two output 
products derived from the NEWSIPS MXHI (i.e. high resolution extracted spectra) files: the "concatenated" spectrum, where the spectral orders are merged eliminating the overlap regions, and the "rebinned" spectrum, which is the concatenated spectrum resampled to the low resolution wavelength step. Both concatenated and rebinned spectra include an error vector calibrated in absolute flux units. The inconsistency between the high resolution short and long wavelength scales in NEWSIPS has been corrected for in the INES concatenated spectra.

In the first part of this paper we discuss the overall quality of NEWSIPS high resolution spectra in terms of accuracy, stability and repeatability of wavelength and flux measurements (Sect. 2). The second part deals with the INES processing of high resolution data, describing the order concatenation and rebinning procedures (Sects. 3.1 and 3.2, respectively). Finally, the application of the correction to the wavelength scale is discussed (Sect. 4).

\section{NEWSIPS data quality evaluation}

The overall quality of $I U E$ high resolution spectra processed with NEWSIPS has been evaluated by studying the accuracy and the stability of wavelength determinations, the accuracy of equivalent width measurements, the flux repeatability and the residual camera non-linearities.

The analysis is based on a large number of spectra, mainly of the $I U E$ standard stars. The spectra have been corrected for the echelle blaze function and calibrated in terms of absolute fluxes according to the procedure described in Paper II. Hereinafter, wavelengths are assumed to be in the heliocentric reference frame and in vacuum.

\subsection{Wavelength accuracy}

To assess the wavelength accuracy three aspects have been considered separately:

a) the accuracy and repeatability of wavelength measurements of a given spectral feature in several spectra of the same star;

b) the stability of the wavelength scale along the full spectral range;

c) the consistency of radial velocity determinations obtained from the SWP, LWP and LWR cameras.

\subsubsection{Expected accuracy}

One of the most important limitations to the wavelength accuracy of IUE high resolution spectra are the target acquisition errors at the nominal center of the spectrographs entrance apertures. These, if large enough, can also affect the quality of the ripple correction (see Paper II).
From the NEWSIPS dispersion constants and the central wavelengths of the spectral orders it can be readily deduced that the velocity dispersion corresponding to one pixel on the image is practically constant all through the range covered by the cameras, and namely:

$$
\begin{aligned}
& \Delta V=7.73 \pm 0.05 \mathrm{~km} \mathrm{~s}^{-1} \text { for SWP } \\
& \Delta V=7.26 \pm 0.09 \mathrm{~km} \mathrm{~s}^{-1} \text { for LWP } \\
& \Delta V=7.26 \pm 0.03 \mathrm{~km} \mathrm{~s}^{-1} \text { for LWR. }
\end{aligned}
$$

Taking into account that the plate scales are 1.530, 1.564 and 1.553 arcsec/pix for SWP, LWP and LWR, respectively (Garhart et al. 1997), an acquisition error of 1 arcsec along the high resolution dispersion direction would lead to a constant velocity offset of $5.1 \mathrm{~km} \mathrm{~s}^{-1}$ for SWP, $4.6 \mathrm{~km} \mathrm{~s}^{-1}$ for LWP and $4.7 \mathrm{~km} \mathrm{~s}^{-1}$ for LWR. Since the pointing/tracking accuracy is usually better than 1 arcsec, we can consider $5 \mathrm{~km} \mathrm{~s}^{-1}$ as a reasonable upper limit to the expected wavelength accuracy. Wavelength errors substantially larger might arise internally in the data extraction procedures.

\subsubsection{Repeatability of wavelength determinations}

To obtain a reliable information on the self-consistency of wavelength determinations, we have attempted to reduce the effects of spectral noise by performing a large number of measurements of selected narrow and symmetric absorption lines from the interstellar medium, which are strong in some of the IUE calibration stars, as well as of many emission lines in RR Tel. Out of the IUE standards, we have selected BD+28 4211, HD 60753, HD 93521, BD $+75325, \lambda$ Lep (HD 34816), $\zeta$ Cas (HD 3360) and $\eta$ UMa (HD 120315). In addition, we have also used spectra of the star $\zeta$ Oph (HD 149757). The present measurements refer only to large aperture spectra.

The interstellar lines selected for the SWP range were: SII $1259.520 \AA$, SiII $1260.412 \AA$, OI $1302.168 \AA$, SiII $1304.372 \AA$ and CII $1334.532 \AA, 1335.703 \AA$. For the long wavelength range we have used the $\mathrm{K}$ and $\mathrm{H}$ components of the MgII doublet at 2796.325 $\AA$ and $2803.530 \AA$ and MgI 2852.965 $\AA$. In the case of $\zeta$ Oph we have also measured MnII 2576.877 $\AA, 2594.507 \AA$ and several FeII lines. The cases in which the distortion of the profile due to close-by reseau marks (in particular for OI 1302 and CII 1334) precluded the accurate determination of the line position have not been taken into account in the final statistics. Laboratory wavelengths have been taken form Morton (1991).

The mean values of the radial velocities, the corresponding rms deviation and the number of independent measurements are reported in Table 1 for each target and camera. According to this table, the rms repeatability error on radial velocities, averaged over the three cameras is $4.6 \pm 1.5 \mathrm{~km} \mathrm{~s}^{-1}$. This value is smaller 
Table 1. Radial velocities obtained from NEWSIPS high resolution spectra

\begin{tabular}{|c|c|c|c|}
\hline Target & SWP & LWP & LWR \\
\hline RR Tel & $-69.5 \pm 6.5[106]$ em. lin. & $-49.3 \pm 3.0[170]$ em. lin. & $-51.0 \pm 4.4[132]$ em. lin. \\
\hline$\zeta \mathrm{Oph}$ & $-24.7 \pm 3.9[60]$ SII, SiII & $-13.4 \pm 2.6[24]$ FeII,MnII & $-10.2 \pm 2.5[69]$ FeII, MnII \\
\hline $\mathrm{BD}+284211$ & $-22.8 \pm 6.4[130]$ SII, SiII, CII & $-5.3 \pm 3.6[40] \mathrm{MgII} \mathrm{K}$ & $-1.1 \pm 4.8[21] \mathrm{MgII} \mathrm{K}$ and $\mathrm{H}, \mathrm{MgI}$ \\
\hline HD 60753 & $18.8 \pm 6.6[62]$ SII, OI, SiII & $32.4 \pm 6.9[70] \mathrm{MgII} \mathrm{K}$ & $29.5 \pm 5.8[24] \mathrm{MgII} \mathrm{K}$ and $\mathrm{H}, \mathrm{MgI}$ \\
\hline HD 93521 & $-38.8 \pm 4.0[68]$ SII, OI, SiII & $-20.7 \pm 6.9[6] \mathrm{MgII} \mathrm{K}$ & $-19.9 \pm 5.4[18] \mathrm{MgII} \mathrm{K}$ and $\mathrm{H}$ \\
\hline $\mathrm{BD}+75325$ & $-16.4 \pm 4.6[121]$ SII, SiII, CII & $6.2 \pm 2.2[43] \mathrm{MgI}$ & $6.6 \pm 3.1[33] \mathrm{MgII} \mathrm{K}$ \\
\hline$\lambda$ Lep & & $21.2 \pm 3.9[37] \mathrm{MgII} \mathrm{K}, \mathrm{MgI}$ & $19.5 \pm 4.9[44] \mathrm{MgII} \mathrm{K}$ and $\mathrm{H}$ \\
\hline$\zeta$ Cas & & $-1.1 \pm 4.2[9] \mathrm{MgII} \mathrm{K}$ & $1.5 \pm 4.4[72] \mathrm{MgII} \mathrm{K}$ and $\mathrm{H}$ \\
\hline$\eta \mathrm{UMa}$ & & $-1.1 \pm 3.1[17] \mathrm{MgII} \mathrm{K}$ & $3.1 \pm 7.1[82] \mathrm{MgII} \mathrm{K}$ and $\mathrm{H}$ \\
\hline
\end{tabular}

The entries for each star are: mean radial velocity, $\mathrm{rms}\left(\mathrm{km} \mathrm{s}^{-1}\right)$, number of measurements, and lines used (em.lin.: emission lines).

All measurements refer to the large aperture.

than the upper limit of about $5 \mathrm{~km} \mathrm{~s}^{-1}$ expected from acquisition errors. Considering the presence of spectral noise, we can safely conclude that the repeatability of wavelength (or velocity) determinations is satisfactory.

The results in Table 1 indicate that the radial velocities derived from the two long wavelength cameras are consistent while, on the contrary, the velocities derived from SWP spectra are systematically more negative. This, and other considerations about the consistency of radial velocity determinations from the three cameras will be discussed in Sect. 4 .

\subsubsection{Stability of the wavelength scale along the full} spectral range

We have studied the accuracy of the wavelength scale over a wide spectral range to look for possible time-dependent distortions across the camera faceplate. To this purpose, we have selected 6 SWP, 11 LWP and 3 LWR spectra of the emission line object RR Tel obtained at different epochs. For each spectrum we have measured the peak wavelengths of several emission lines chosen among those reasonably well exposed and with the cleanest profiles, covering the full spectral range. The highest excitation lines, such as those from $[\mathrm{MgV}]$, were purposely excluded because they provided systematically higher negative radial velocities probably due to stratification effects within the nebular region. The mean radial velocities of $\mathrm{RR}$ Tel are $-69.5 \pm 6.5 \mathrm{~km} \mathrm{~s}^{-1}$, (SWP), $-49.3 \pm 3.0 \mathrm{~km} \mathrm{~s}^{-1}$ (LWP), and $-51.0 \pm 4.1 \mathrm{~km} \mathrm{~s}^{-1}$ (LWR). The total number of measurements are 106, 170 and 132 for SWP, LWP and LWR, respectively. Since the errors are of the same order as the repeatability errors quoted in the previous section, we conclude that the wavelength scales do no present appreciable distortions over the wavelength range covered and, within the observational errors, are stable over the period of time considered (1983-1994, 1985-1995 and 1978-1983 for SWP, LWP and LWR, respectively).
2.1.4. Radial velocity determinations from the $\mathrm{Mg}$ II doublet

The present analysis has revealed the existence of an inconsistency in the radial velocities derived from the MgII $K(2796.32 \AA)$ and $H(2803.53 \AA)$ lines as measured in the LWP camera, where the two lines are present in both orders 82 and 83 . To quantify this discrepancy, we have measured the velocities of the Mg II interstellar lines in 89 spectra of five $I U E$ standard stars.

We find that, in the LWP camera, the radial velocity difference $V_{\mathrm{H}}-V_{\mathrm{K}}$ is $-10.8 \pm 2.5 \mathrm{~km} \mathrm{~s}^{-1}$ when measured in order 83 , and $11.7 \pm 1.5 \mathrm{~km} \mathrm{~s}^{-1}$ when measured in order 82 . We find also that there is a discrepancy in the velocity of the $K$ line measured in the two orders $\left(V_{83}-V_{82}=-20.5 \pm 0.9 \mathrm{~km} \mathrm{~s}^{-1}\right)$, while the velocities of the $H$ line measured in the two orders are consistent within $2 \mathrm{~km} \mathrm{~s}^{-1}$, on average. Since the velocity derived from other interstellar lines (e.g. MgI 2852.965 ̊) is fully consistent with the measurements from the $K$ line in order 83 , we conclude that only this line provides correct radial velocity values. In INES concatenated spectra (Sect. 3.1) the $K$ line comes from order 83 and the $H$ line from order 82 . Therefore, there is a systematic difference $V_{\mathrm{K}}-V_{\mathrm{H}}=-8.8 \pm 1.3 \mathrm{~km} \mathrm{~s}^{-1}$ between the velocities determined from the two lines, being the correct value only that given by the $K$ line.

A similar study performed in LWR spectra, where the $K$ line appears only in order 83 , shows that this problem is not present in this camera, where the two Mg II lines provide consistent velocity values: $V_{\mathrm{K}}($ order 83$)-$ $V_{\mathrm{H}}($ order82 $)=-1.0 \pm 1.2 \mathrm{~km} \mathrm{~s}^{-1}$.

\subsection{Background extraction}

It has been repeatedly pointed out that the background extraction for high resolution spectra processed with IUESIPS was not accurate enough especially shortward of $1400 \AA$ in the SWP camera and $2400 \AA$ in the long wavelength cameras, as denoted by the negative fluxes 


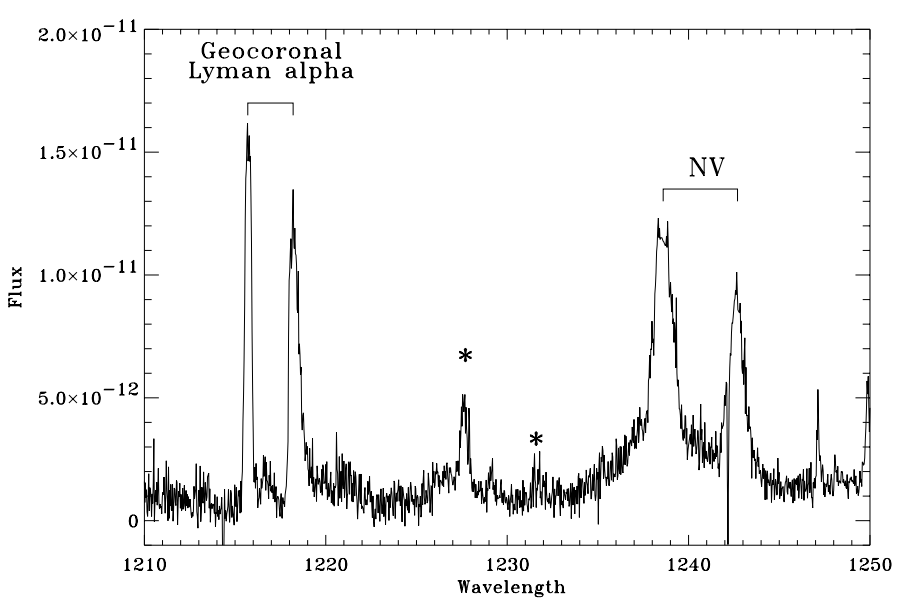

Fig. 1. A portion of the longest available SWP exposure of RR Tel (SWP20246: 820 minutes). The two Lyman $\alpha$ lines arise from geocoronal emission filling the large and the small apertures. The emission features labeled with an asterisk are due to overspilling of the strong NV emission into the adjacent order

assigned to the wings of the strongest emission lines and to the core of the saturated absorption lines. As shown below, this effect is not present anymore in spectra processed with NEWSIPS, which makes use of an upgraded background determination procedure (Smith 1999).

Overestimating or underestimating the background level leads to underestimating or overestimating the fluxes of the emission lines and the equivalent widths of the absorption lines. In the following, we report the tests done on the accuracy of the equivalent widths to verify the correctness of the background extraction. In addition, we have used the repeatability of the equivalent widths determinations as an indirect test of the stability of the background levels.

\subsubsection{SWP}

Figure 1 shows the profiles of the NV doublet emission and of the broad Lyman $\alpha$ feature in the longest exposure available of RR Tel. It is clearly seen that the wings of these lines are not assigned negative values. Particularly interesting are the NV "ghost" lines marked with an asterisk, which are still present in NEWSIPS data, but sensibly fainter than in the IUESIPS spectra, most likely due to the optimized extraction slit. The presence of such spurious lines has recently been reported by Zuccolo et al. (1997) and ascribed to overspilling of the strong NV doublet into adjacent orders.

To verify the accuracy of the background subtraction, we have compared the equivalent widths of the strongest interstellar lines in four spectra of $\zeta$ Oph with those reported by Morton (1975) obtained from Copernicus data. These latter determinations are presumably not affected by background determination problems, unlike the $I U E$

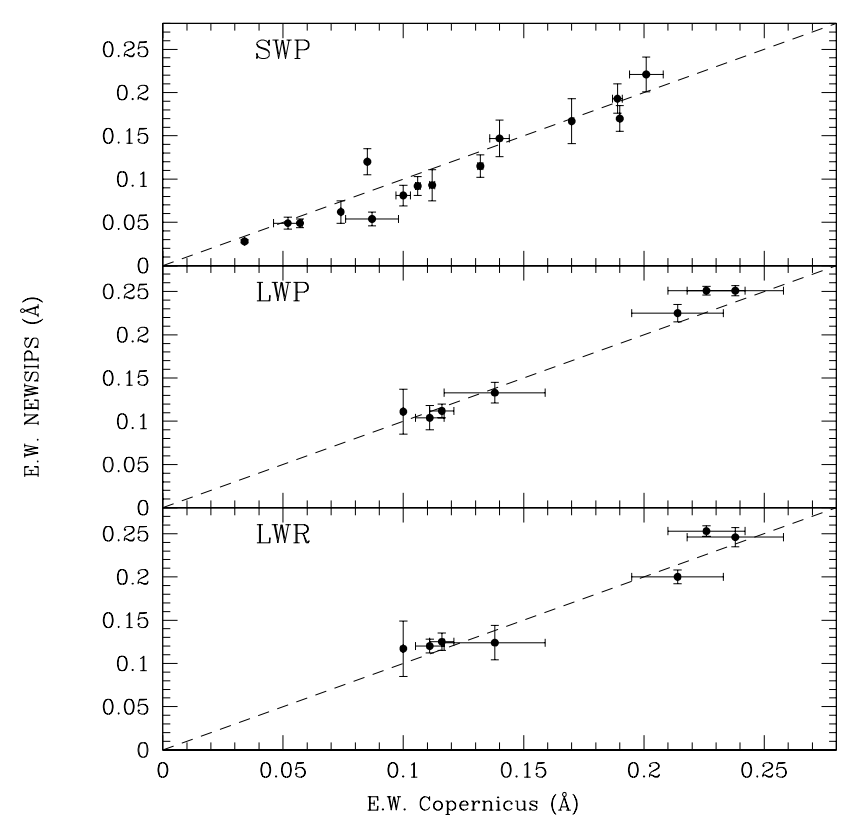

Fig. 2. Comparison of the equivalent widths of interstellar lines in the spectrum of $\zeta$ Oph as measured in Copernicus and in NEWSIPS spectra. Copernicus measurements have been taken from Morton (1975). The dashed line in each panel indicates the $1: 1$ relation

Table 2. Equivalent widths (in $\mathrm{m} \AA$ ) in SWP spectra of $\zeta$ Oph compared with Copernicus measurements

\begin{tabular}{lcl}
\hline Line & NEWSIPS & Morton (1975) \\
\hline SII 1250.59 & $81 \pm 12$ & $100 \pm 3$ \\
SII 1253.81 & $92 \pm 11$ & $106 \pm 2$ \\
SII 1259.52 & $93 \pm 18$ & $112 \pm 1$ \\
SiII 1260.42 & $167 \pm 26$ & 70 \\
CI 1277.24 & $62 \pm 13$ & 74 \\
OI 1302.17 & $221 \pm 20$ & $201 \pm 7$ \\
SiII 1304.37 & $115 \pm 13$ & $132 \pm 1$ \\
CI 1328.83 & $49 \pm 7$ & $52 \pm 6$ \\
CII 1334.53 & $193 \pm 17$ & $189 \pm 2$ \\
CII 1335.70 & $147 \pm 21$ & $140 \pm 4$ \\
SiII 1526.71 & $170 \pm 15$ & $190:$ \\
FeII 1608.46 & $120 \pm 15$ & $85:$ \\
SiII 1808.01 & $54 \pm 8$ & $87 \pm 11$ \\
AlIII 1854.72 & $49 \pm 5$ & $57:$ \\
AlIII 1862.79 & $28 \pm 2$ & $34:$ \\
\hline
\end{tabular}

: Means uncertain value.

echelle spectra near the short wavelength end of the cameras. The results of the comparison are given in Fig. 2 and in Table 2, which provides the mean and the standard deviation of the four measurements. As it appears clearly from the figure, the NEWSIPS measurements are consistent, within the errors, with the values from Copernicus data, suggesting that the background evaluation for SWP spectra is essentially correct. 
Table 3. Equivalent widths (in $\mathrm{m} \AA$ ) in LWP and LWR spectra of $\zeta$ Oph compared with Copernicus measurements

\begin{tabular}{llll}
\hline Line & \multicolumn{1}{c}{ LWP } & \multicolumn{1}{c}{ LWR } & Morton (1975) \\
\hline ZnII 2026.16 & $111 \pm 26$ & $117 \pm 32$ & $100:$ \\
FeII 2382.76 & $251 \pm 6$ & $246 \pm 11$ & $238 \pm 20$ \\
MnII 2576.88 & $133 \pm 12$ & $124 \pm 20$ & $138 \pm 21$ \\
FeII 2586.65 & $225 \pm 10$ & $200 \pm 8$ & $214 \pm 19$ \\
MnII 2594.51 & $112 \pm 8$ & $125 \pm 10$ & $116 \pm 5$ \\
FeII 2600.17 & $251 \pm 5$ & $253 \pm 6$ & $226 \pm 16$ \\
MnII 2606.48 & $104 \pm 14$ & $120 \pm 8$ & $111 \pm 6$ \\
\hline
\end{tabular}

: Means uncertain value.

Table 4. Repeatability of equivalent widths measurements SWP

\begin{tabular}{lcc}
\hline Line & BD +284211 & BD +75325 \\
\hline SII 1250.59 & $32 \pm 9(29)$ & $34 \pm 8(26)$ \\
SII 1259.52 & $48 \pm 10(28)$ & $70 \pm 12(35)$ \\
SII 1260.42 & $92 \pm 12(37)$ & $128 \pm 14(25)$ \\
CII 1334.53 & $115 \pm 14(36)$ & $125 \pm 19(35)$ \\
\hline \multicolumn{3}{c}{ LWP } \\
\hline Bine +284211 & BD +75325 \\
\hline MgII 2796.53 $257 \pm 20(37)$ & $369 \pm 50(33)$ \\
MgI 2852.97 & $276 \pm 40(11)$ & $45 \pm 16(10)$ \\
\hline \multicolumn{3}{c}{ LWR } \\
\hline Line UM $\pm \zeta$ Cas \\
\hline MgII 2796.35 \\
MgII 2803.53 $\AA$ & $65 \pm 11(41)$ & $354 \pm 22(36)$ \\
\hline
\end{tabular}

Equivalent widths in $\mathrm{m} \AA$. The number of measurements is given in brackets.

The stability of the background subtraction has been evaluated by measuring the equivalent width of several strong interstellar lines in a large sample of spectra of two standard stars. The repeatability of the equivalent widths ranges from $10 \%$ for the strongest lines to $30 \%$ for the faintest ones (Table 4).

\subsubsection{LWP}

We tested the accuracy of the background extraction from the cores of strongly saturated absorption lines and the wings of strongly saturated emission lines. Figure 3 shows a portion of a spectrum of SN 1987A centered around the $\mathrm{Mg}$ II doublet at $2800 \AA$. It appears from the figure that the cores of the absorption lines do not become systematically negative, as expected for a correct background extraction. Shown in the same figure is another example, that of the strongly saturated Mg II emission doublet in the longest LWP exposure available of RR Tel (LWP25954): the lines wings do not reach negative fluxes, as required.

A second test has been performed in four spectra of $\zeta \mathrm{Oph}$, in which we have measured the equivalent widths
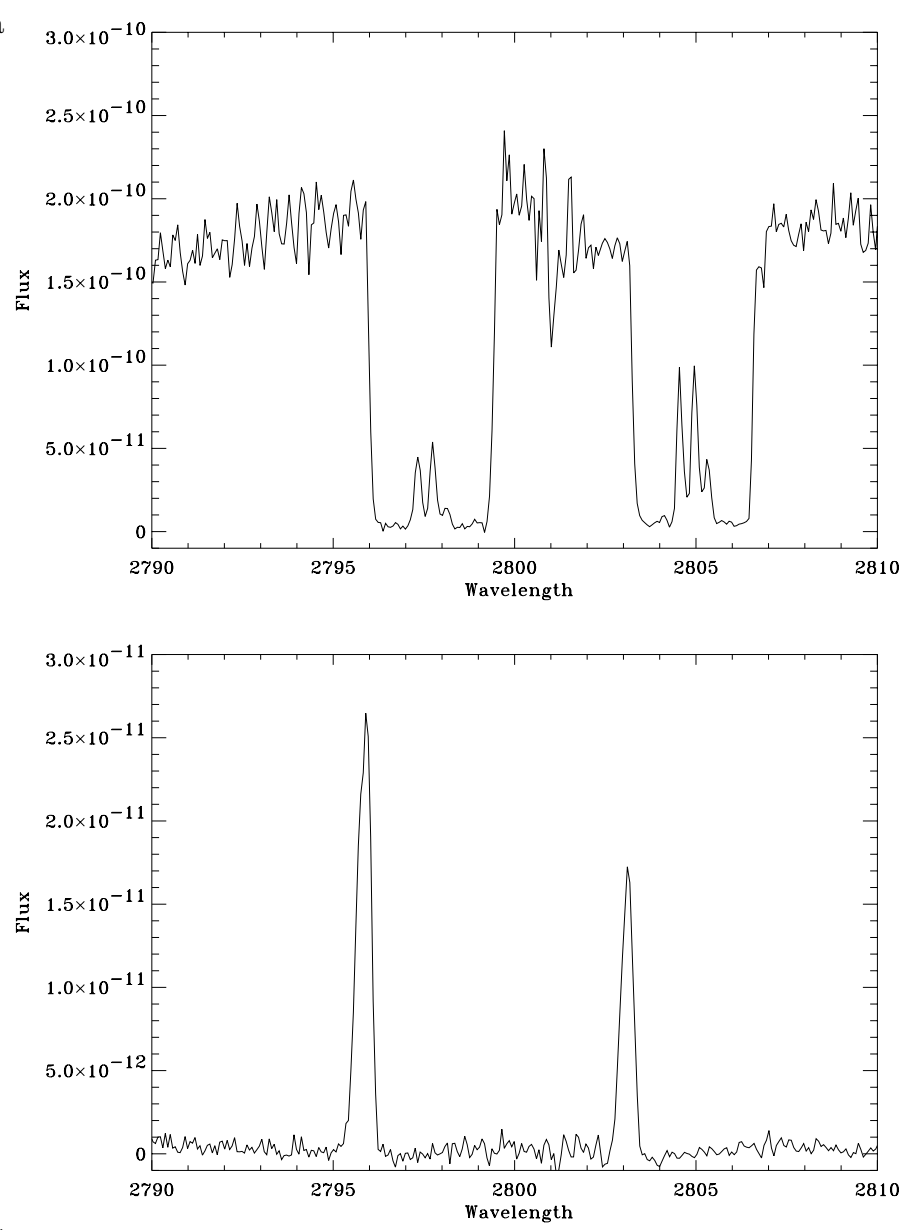

Fig. 3. Top panel: The region of the MgII doublet in the spectrum of SN 1987A (LWP10194). Bottom panel: The same region in the deepest LWP exposure of RR Tel (LWP25954)

of some strong interstellar lines, and compared them with the Copernicus values given by Morton (1975). The results are summarized in Fig. 2 and Table 3, where are given the mean and the standard deviation of the four spectra.

Finally, we have measured the equivalent widths of the MgII and MgI lines in a large sample of spectra of $\mathrm{BD}+284211$ and $\mathrm{BD}+75325$. The repeatability errors range from $35 \%$ for the faint $\mathrm{MgI}$ line in $\mathrm{BD}+75325$, to less than than $10 \%$ for the strong lines. Results are shown in Table 4.

\subsubsection{LWR}

As for the LWR camera, we have verified the accuracy of the background subtraction by measuring the equivalent widths of six strong FeII and MnII interstellar lines in four spectra of $\zeta \mathrm{Oph}$, and compared them with measurements based on Copernicus data. As shown in Fig. 2, there is a good agreement between the two sets of equivalent widths, and no systematic departures are found. 
Table 5. Flux repeatability of high resolution spectra SWP

\begin{tabular}{lccc}
\hline Band & Order & set A & set B \\
\hline $1185-1190$ & 116 & 3.2 & 3.5 \\
$1225-1230$ & 112 & 2.3 & 4.3 \\
$1285-1290$ & 107 & 2.6 & 3.2 \\
$1375-1380$ & 100 & 1.8 & 3.0 \\
$1485-1490$ & 93 & 1.6 & 3.9 \\
$1595-1600$ & 86 & 3.5 & 3.5 \\
$1795-1800$ & 77 & 1.6 & 2.5 \\
\hline
\end{tabular}

Set A: BD+28 4211: a) 4 spectra (Jun. 86-Dec. 86); b) 6 spectra (Nov. 90-Jul. 91), BD+75 325: 8 spectra (Dec. 88-May 89), HD 60753: a) 4 spectra (Apr. 80-May 80); b) 8 spectra (Feb. 88-May 89), HD 93521: 11 spectra (March 1987).

Set B: BD+28 4211: 45 spectra (Dec. 82-Aug. 95), BD+75 425: 53 spectra (Sep. 85-Feb. 95), HD 60753: 38 spectra (Jul. 79March 95).

LWP

\begin{tabular}{lccc}
\hline Band & Order & set A & set B \\
\hline $2117-2122$ & 109 & 4.9 & 6.3 \\
$2457-2462$ & 94 & 2.0 & 3.5 \\
$2922-2927$ & 79 & 1.8 & 2.7 \\
$3117-3122$ & 74 & 3.0 & 4.0 \\
\hline
\end{tabular}

Set A: BD+75 325: a) 11 spectra (March 85-Dec. 85); b) 9 spectra (Jan. 89-Dec. 89); c) 8 spectra (Feb. 92-Nov. 92).

Set B: BD+28 4211: 34 spectra (Dec. 82-Aug. 95), BD+75 325: 37 spectra (Sep. 85-Feb. 95), HD 60753: 24 spectra (Jul. 86Aug. 95).

LWR

\begin{tabular}{lccc}
\hline Band & Order & Set A & Set B \\
\hline $2117-2122$ & 109 & 4.2 & 5.5 \\
$2457-2462$ & 94 & 3.4 & 6.3 \\
$2922-2927$ & 79 & 1.8 & 4.3 \\
$3077-3082$ & 75 & 2.4 & 5.0 \\
\hline
\end{tabular}

Set A: $\zeta$ Cas: a) 4 spectra (Dec. 81-Dec. 82); b) 5 spectra (Sep. 83-Jul. 84), $\eta$ UMa: a) 6 spectra (March 81-Apr. 82); b) 5 spectra (Aug. 82-Jun. 86).

Set B: $\zeta$ Cas: 30 spectra (Feb. 81-Feb. 87), $\eta$ UMa: 42 spectra (Sep. 78-Jul. 90).

The repeatability of equivalent width determinations has been determined measuring the equivalent widths of the MgII doublet in a large sample of spectra of $\eta \mathrm{UMa}$ and $\zeta$ Cas and $\lambda$ Lep. The repeatability error ranges from $30 \%$ for the weak lines to less than $5 \%$ for the strongest ones (Table 4).

\subsection{Flux repeatability}

Two different tests have been performed to assess the flux repeatability of high resolution spectra. In the first one we selected restricted samples of spectra obtained close in time, and measured the ripple corrected net fluxes, i.e. without applying the time sensitivity degradation and the temperature dependence corrections. The second test has been performed on larger samples covering extended periods of time, measuring the absolutely calibrated fluxes, which include time and temperature corrections. In all case we have averaged the flux over a narrow wavelength interval free of lines. The flux repeatibility was defined as the percent rms deviation from the mean value. The results are summarized in Table 5 .

\subsubsection{SWP}

For the first test we have used 41 high resolution spectra of IUE calibration standards grouped into sets of data with a similar exposure level and obtained close enough in time. The test was done in six bands $5 \AA$ wide. In Table 5 (under "set A") we report the percent rms deviation. The repeatability of spectra obtained sufficiently close in time is about $2 \%$. The second test was made on a larger number of spectra with similar exposure time, without restricting the date of observation ("set B"). This test provides repeatability errors ranging from 3 to $4 \%$. These somewhat larger errors are due to the intrinsic uncertainties of the sensitivity degradation correction algorithm.

\subsubsection{LWP}

The tests performed are similar to those described above for the SWP camera. The spectra in "Set A" consist of three groups each containing images obtained in a restricted period of time. The flux repeatability was evaluated in four wavelength bands $5 \AA$ wide. In this camera, the repeatability errors can reach the $5 \%$ level near the short wavelength end of the camera, but are a factor of two lower in the central bands. A similar test performed on a larger set of spectra needing correction for the time-dependent sensitivity degradation ("Set B") provides errors slightly larger, confirming that the sensitivity degradation algorithm adopted for the LWP camera is essentially correct.

\subsubsection{LWR}

The flux repeatability was evaluated in four wavelength bands $5 \AA$ wide. The test performed on spectra taken close in time, "Set A", shows that the repeatability errors reach $4 \%$ near the short wavelength end of the camera, decreasing in the region of maximum sensitivity and increasing again at the longest wavelengths. In the "Set B" spectra the repeatability is worse, reflecting the uncertainties in the time degradation correction and also the instability of the camera after it ceased to be routinely used. 
Table 6. Linearity study for high resolution spectra

SWP

\begin{tabular}{|c|c|c|c|c|c|}
\hline Image & $t / t(\mathrm{opt})$ & $1185 \AA$ & $1285 \AA$ & $1485 \AA$ & $1785 \AA$ \\
\hline 41467 & 0.50 & 0.94 & 0.95 & 0.97 & 1.05 \\
\hline 41346 & 0.68 & 0.92 & 0.98 & 0.95 & 1.02 \\
\hline 42309 & 0.70 & 0.97 & 0.93 & 0.98 & 1.00 \\
\hline 41435 & 0.90 & 0.96 & 0.99 & 1.01 & 0.99 \\
\hline 41466 & 1.00 & 0.98 & 0.98 & 1.00 & 0.98 \\
\hline 42260 & 1.00 & 1.02 & 1.02 & 1.01 & 1.03 \\
\hline 41495 & 1.80 & 0.96 & 0.98 & 0.98 & 0.99 \\
\hline \multicolumn{6}{|c|}{ LWP } \\
\hline Group & $t / t(\mathrm{opt})$ & $2120 \AA$ & $2460 \AA$ & $2925 \AA$ & $3132 \AA$ \\
\hline $\mathrm{a}$ & 0.27 & 1.03 & 1.03 & 0.98 & 0.98 \\
\hline $\mathrm{b}$ & 0.41 & 1.00 & 1.02 & 0.99 & 0.98 \\
\hline $\mathrm{c}$ & 0.67 & 0.99 & 1.02 & 1.03 & 1.00 \\
\hline d & 0.83 & 0.95 & 0.98 & 1.00 & 0.97 \\
\hline e & 1.00 & 1.00 & 1.00 & 1.00 & 1.00 \\
\hline $\mathrm{f}$ & 1.33 & 0.93 & 1.03 & 1.00 & 1.03 \\
\hline $\mathrm{g}$ & 2.07 & 1.01 & 1.04 & 1.04 & 1.08 \\
\hline
\end{tabular}

a: 2 spectra of $\mathrm{BD}+284211$,

b: 1 spectrum of $\mathrm{BD}+284211$,

c: 2 spectra of $\mathrm{BD}+284211$,

d: 2 spectra of $\mathrm{BD}+75325$,

e: reference group: 4 spectra of $\mathrm{BD}+75325$ and 4 spectra of $\mathrm{BD}+284211$,

f: 1 spectrum of $\mathrm{BD}+75325$,

g: 1 spectrum of $\mathrm{BD}+75325$.

LWR

\begin{tabular}{lccccc}
\hline Image & $t / t(\mathrm{opt})$ & $2120 \AA$ & $2460 \AA$ & $2925 \AA$ & $3080 \AA$ \\
\hline 9955 & 0.50 & 0.75 & 0.98 & 0.99 & 0.91 \\
9954 & 1.00 & 1.00 & 1.00 & 1.00 & 1.00 \\
9113,9953 & 2.25 & 1.18 & 1.05 & 1.00 & 1.01 \\
8116 & 2.50 & 1.12 & 1.04 & 1.01 & 1.00 \\
\hline
\end{tabular}

\subsection{Flux linearity}

Despite the linearity correction applied during the processing, residual non linearities are still present in $I U E$ data. This effect has been evaluated in Paper I for low resolution data. In what follows we discuss this effect in high resolution spectra. The method followed consists on studying a set of spectra of the same star with different exposure times obtained, whenever possible, very close in time (preferably on the same observing shift) and with similar camera temperatures. The variation of the flux with the level of exposure (or the exposure time) defines the flux linearity. Unfortunately there exist few sets of high resolution data suitable for this study, and a slightly different approach has been taken here. The results are summarized in Table 6. In general, these results are in good agreement with those derived in Paper I.

\subsubsection{SWP}

The most complete set of SWP spectra appropriate to assess the linearity consists of seven images of the white dwarf CD-38 10980 obtained in the period April-August 1991, with exposure times ranging from $50 \%$ to $180 \%$ of the optimum value $(200 \mathrm{~min})$. For these images we have measured the mean flux in five $5 \AA$ wide bands. The fluxes in each band have been averaged together and divided by the mean flux in the two $100 \%$ exposures. The ratios so obtained indicate departures from linearity ranging from $-6 \%$ at $1185 \AA$ to $+4 \%$ at $1785 \AA$ for the $50 \%$ exposure and up to $-5 \%$ for the $70 \%$ exposure.

\subsubsection{LWP}

There is not any complete set of high resolution data of the same star which allows to study the LWP camera linearity. We have constructed average spectra of different exposure levels of the two standard stars BD+28 4211 and $\mathrm{BD}+75325$, and divided them by the corresponding $100 \%$ spectrum. The exposure levels covered range from $27 \%$ to $207 \%$ of the optimum exposure time. The test was performed in four wavelength bands $5 \AA$ wide, selected for being relatively free from strong absorption lines. The maximum departures from linearity, reaching $8 \%$, are found for the $133 \%$ level at $2120 \AA$, and for the $207 \%$ level near the regions of maximum sensitivity of the camera. The latter deviation can be easily understood in terms of saturation.

2.4.3. LWR

The LWR high resolution linearity test has been performed with five images of the standard star HD 93521 obtained in the period July 1980 to February 1981, covering the range of exposure times from $50 \%$ to $250 \%$ of the optimum value. The test was performed in four bands $5 \AA$ wide. The maximum departures form linearity (up to $25 \%$ ) are found at the shortest wavelengths, where fluxes are underestimated by $25 \%$ for the $50 \%$ exposure and overestimated by $12 \%$ for the $250 \%$ exposure.

\section{INES processing of high dispersion spectra}

The starting point for the INES processing of high resolution data are the NEWSIPS MXHI files. The spectra have not been re-extracted from the bi-dimensional files, as in the case of the low resolution data (see Paper I). The INES system provides two output spectra for each high dispersion image: the "concatenated" and the "rebinned" spectra. Both include a modified wavelength scale and the error vector calibrated in absolute flux units. All these aspects are discussed in the following sections. 


\subsection{The high resolution concatenated spectra}

The main features of the INES concatenated spectra are:

a) The overlap regions between adjacent orders are suppressed in such a way that the less noisy portion of the orders is retained.

b) The error vector is calibrated in absolute flux units.

c) The wavelength scale is modified to make consistent the radial velocities obtained from the short and the long wavelength cameras. The wavelength sampling of the MXHI files has been retained.

d) The spectra are provided as FITS tables having the same format as the low resolution spectra, i.e. they contain only four columns: wavelength, absolute flux, error and quality factor. This setting reduces significantly the download time for remote data retrieval, and simplifies considerably the structure of the NEWSIPS MXHI files, which contain additional information, not relevant for most investigations, such as the position of the orders on the bi-dimensional image, the height of the extraction slit and the order number for each extracted point.

The procedure followed to concatenate the spectral orders is described in detail in the next paragraphs.

\subsubsection{The concatenation procedure}

The most critical point in the concatenation of adjacent echelle orders is a suitable definition of the "cut wavelengths" so that only the highest quality data points of the overlap region are retained.

In IUE high resolution spectra, the signal-to-noise level at the edge of the orders is different in the short and long wavelength cameras. In the LWP and LWR cameras the signal-to-noise is always lower at the short wavelength end of the orders (except in the highest orders, see below). The contrary happens in the SWP camera, where the long wavelength edge of the orders is much noisier than the short wavelength one. Taking this into account, the cut wavelengths have been defined as follows:

SWP:

$\lambda_{\text {cut }}=\lambda_{\text {start }}+\left(\lambda_{\text {end }}-\lambda_{\text {start }}\right) / 3$.

LWP/LWR:

$\lambda_{\text {cut }}=\lambda_{\text {start }}+2 \times\left(\lambda_{\text {end }}-\lambda_{\text {start }}\right) / 3$.

where

$\lambda_{\text {cut }}$ : cut wavelength between orders $m$ and $m-1$

$\lambda_{\text {start }}$ : start wavelength of overlap region (order $m-1$ )

$\lambda_{\text {end }}$ : end wavelength of overlap region (order $m$ ).

These expressions are valid for all spectral orders except order 125 in the LWP camera and orders 120 to 125 in the LWR camera, where the $\mathrm{S} / \mathrm{N}$ ratio in order $m$ is systematically higher than in order $m-1$ in the overlap region. In these cases only the points of order $m$ are taken.

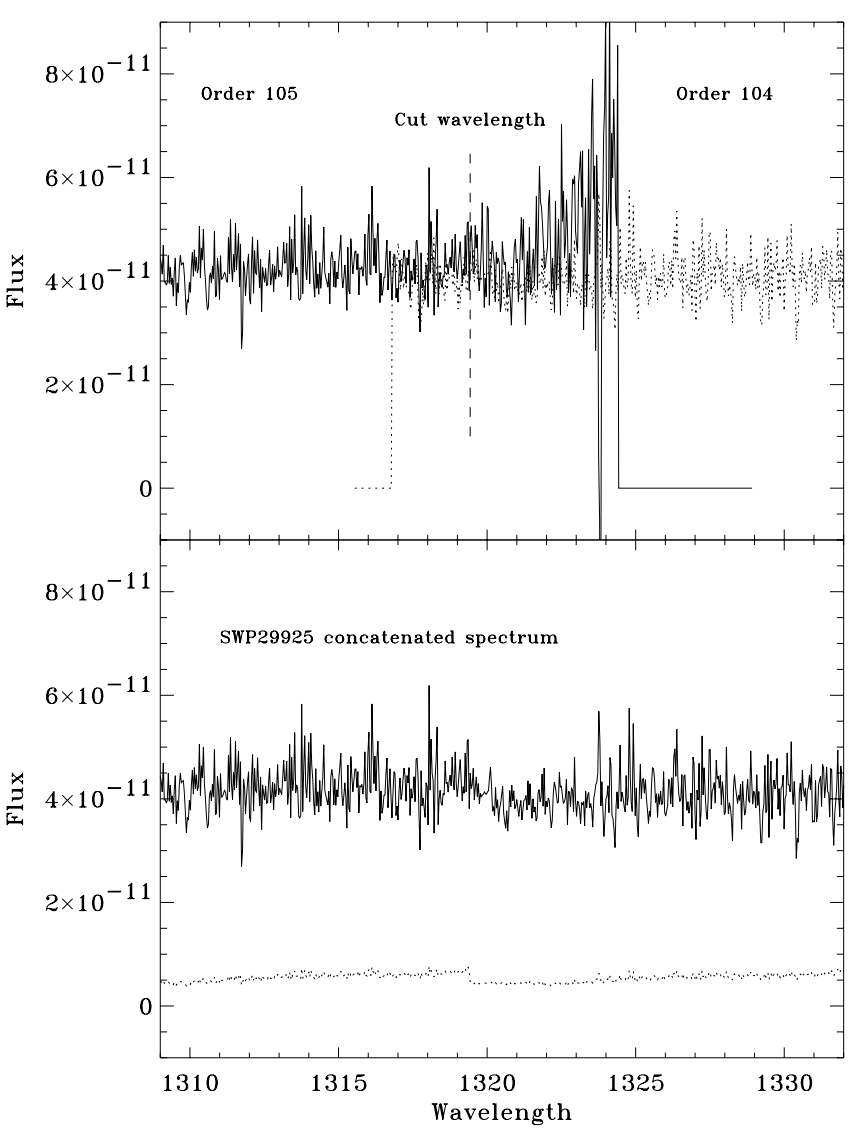

Fig. 4. Example of the concatenation procedure for the SWP camera. The top panel shows the individual orders from the MXHI file. The bottom panel shows the final concatenated spectrum and the calibrated errors (dotted line)

The above defined cut wavelengths (i.e. end wavelengths of order $m$ ) can be computed as a function of order number as:

$\lambda_{\text {cut }}(m)=A+\frac{B}{m}+\frac{C}{m^{2}}$

with the values of $A, B$ and $C$ given in Table 7 .

For non overlapping orders (lower than 73, 77 and 76 for SWP, LWP and LWR, respectively) only photometrically corrected pixels have been included in the concatenated spectra (see Fig. 6). The concatenated spectra cover the same spectral range as the INES low resolution spectra, i.e. $1150-1980 \AA$ for the SWP camera, and $1850-3350 \AA$ for LWP and LWR.

Figures 4, 5 and 6 show examples of the concatenation procedure for the three cameras.

\subsubsection{The error vector}

The NEWSIPS processing provides an error vector for the high resolution spectra which is computed simply as the sum along the extraction slit of the noise values for 


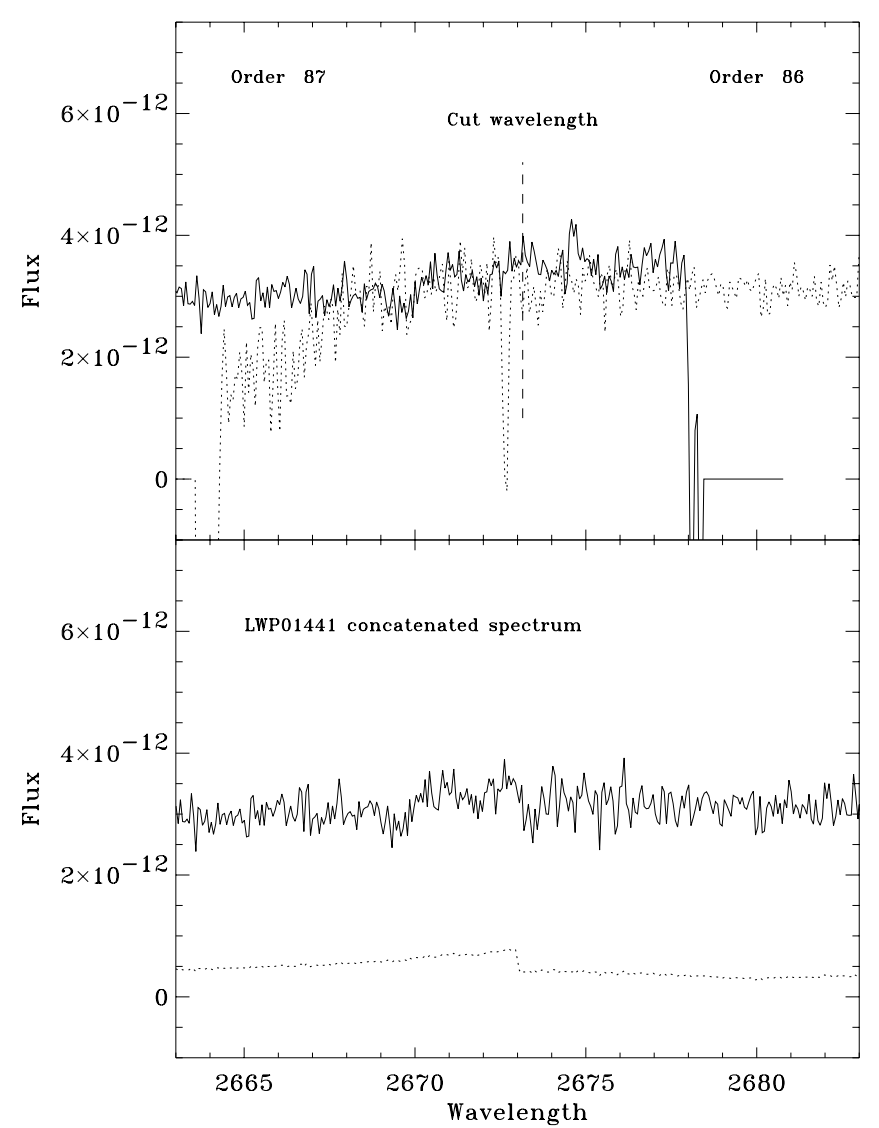

Fig. 5. As Fig. 4 for the LWP camera

Table 7. Parameters defining the cut wavelengths for the order concatenation

\begin{tabular}{rcccc}
\hline Camera & $\mathrm{A}$ & $\mathrm{B}$ & $\mathrm{C}$ & Orders \\
\hline SWP LAP & 24.3952 & 132875.4838 & 325840.9715 & $120-73$ \\
SAP & 22.2095 & 133293.4862 & 300351.2209 & $120-73$ \\
LWP LAP & -7.9697 & 233257.6280 & 0 & $124-77$ \\
SAP & -7.7959 & 233382.6450 & 0 & $124-77$ \\
LWR LAP & -11.3459 & 233737.5903 & 0 & $119-76$ \\
SAP & -11.2214 & 233876.9950 & 0 & $119-76$ \\
\hline
\end{tabular}

the individual pixels, as derived from the camera noise model. Unlike the "sigma" of the low resolution data, the "sigma" vector in the MXHI files is not flux calibrated but given in FN (Flux Number) units.

In the INES high resolution data, the "sigma" spectrum is provided in absolute flux units. The calibration is performed by applying to the MXHI error vector the high resolution calibration and the time sensitivity degradation correction.

\subsection{The resampled spectra}

In the INES Archive, each high resolution image has an associated "rebinned" spectrum, which is obtained by rebinning the "concatenated" spectrum at the same wavelength step size as low resolution data. This data set

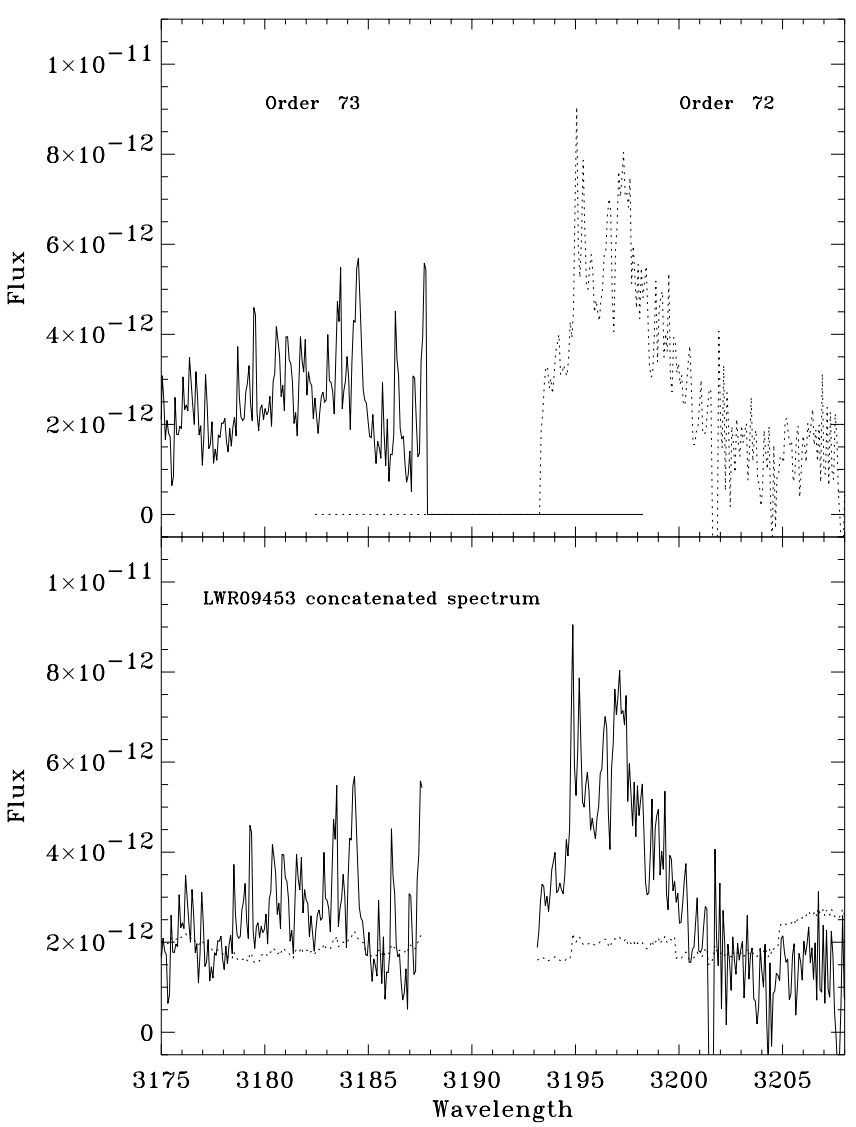

Fig. 6. As Fig. 4 for the LWR camera. In the spectral region shown orders do not overlap. The INES concatenated spectrum has no data in that region

represents an important complement to the low resolution archive, and it is especially useful for time variability studies. The rebinned spectra have not been convolved with the low resolution Point Spread Function, and therefore have a better spectral resolution than low dispersion spectra. Examples of rebinned spectra are shown in Figs. 7 and 8.

The high resolution concatenated spectra (derived as described in the previous section) are resampled into the low resolution wavelength space following the procedure detailed below.

\subsubsection{The rebinning procedure}

The concatenated spectra have been resampled into the INES low resolution wavelength domain as defined in Paper I. The sampling interval is $1.6764 \AA /$ pixel and $2.6693 \AA /$ pixel, for the short wavelength and the long wavelength ranges, respectively, and the wavelength coverage is $1150-1980 \AA$ for SWP and $1850-3350 \AA$ for LWP and LWR. 


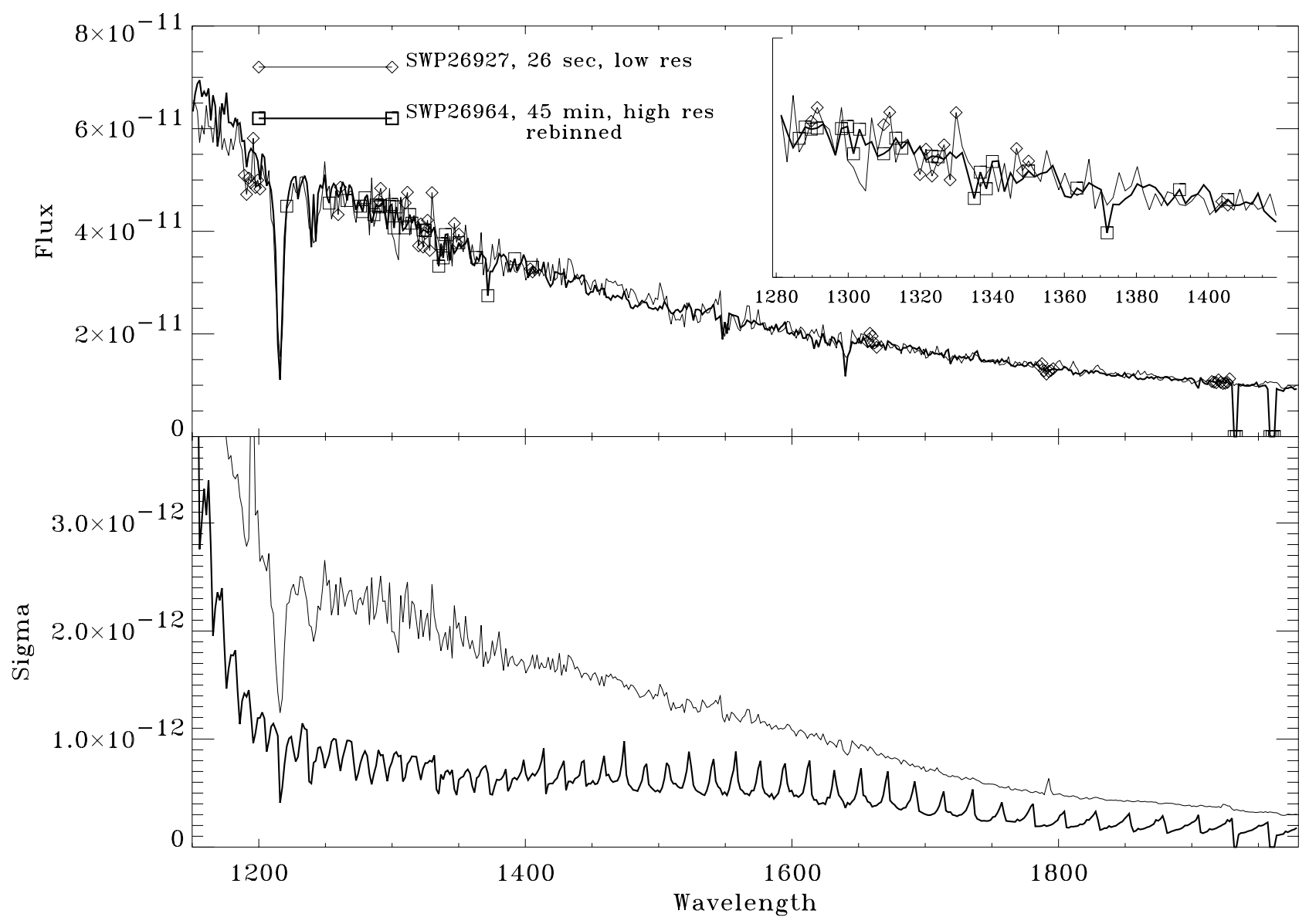

Fig. 7. Comparison of low resolution (thin line) and high resolution rebinned (thick line) spectra of the standard star BD +284211 . The top panel shows the flux spectra. Flagged pixels are marked with diamonds (low resolution) and squares (rebinned). The two gaps in the high resolution spectra longward $1900 \AA$ correspond to the regions where spectral orders do not overlap, which have been assigned zero flux. The inset in the top panel shows in more detail the region $1300-1400 \AA$. The error spectrum is shown in the bottom panel (thin line: low resolution, thick line: rebinned). The characteristic pattern of the errors of the rebinned spectrum is due to the lower signal-to-noise ratio at the edges of the individual echelle orders

The resampling has been performed so that the total flux is conserved, that is, if $n$ pixels with fluxes $f_{1}, f_{2}, \cdots$, $f_{n}$ are rebinned into one, the total flux in the bin is:

$F=\sum_{i=1}^{i=n}\left(\lambda_{i}-\lambda_{i-1}\right)\left(f_{i}+f_{i-1}\right) / 2$

where the flux at the bin edges $(i=1, i=n)$ is calculated by linearly interpolating between the two adjacent pixels. The flux of the final pixel is:

Flux $=F /$ step

being "step" the low resolution pixel size defined above.

\subsubsection{Errors}

The rebinned error spectrum is computed from the concatenated error spectrum according to the following expression:

$E=\frac{\sqrt{\sum e_{i}^{2}}}{n}$

where $e_{i}$ are the errors of the original pixels in the concatenated spectrum.

\subsubsection{Flagging}

The quality flag assigned to each pixel in the resampled spectrum is the sum of the flags of the original high resolution pixels. Only the most relevant quality flags present in the high resolution spectrum have been transmitted to 


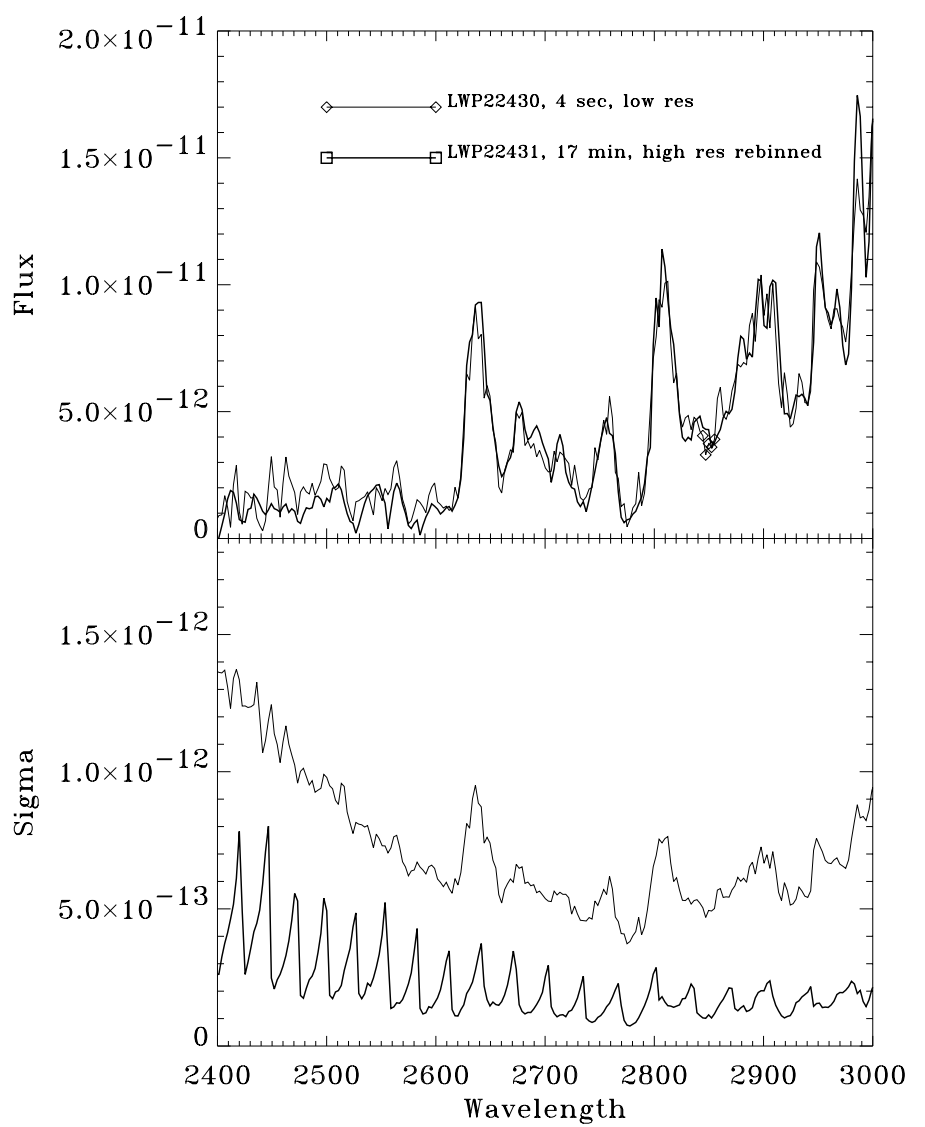

Fig. 8. Similar to Fig. 7 but comparing two LWP spectra of Nova Cygni 1992. The errors are shown in the bottom panel

the rebinned spectrum:

-8192: Missing minor frames in extracted spectrum,

-1024: Saturated pixel,

-16: Microphonic noise (for the LWR camera only),

-8: Potential DMU corrupted pixel,

-2: Uncalibrated data point.

Other flags (e.g. reseau marks) have not been taken into account to avoid that a too large fraction of the pixels in the output spectrum come out flagged with error conditions, despite their quality is not significantly affected. Pixels corresponding to the gaps between non-overlapping orders are flagged with " -2 ".

\section{The correction to the wavelength scale}

We already pointed out in Sect. 2 that there is a significant discrepancy between the wavelength scales of short and long wavelength spectra processed with NEWSIPS. This inconsistency, which is well above the accuracy of the wavelength calibration, was already present in IUESIPS data (Nichols-Bohlin \& Fesen 1986, 1990).

Table 8 presents a summary of the radial velocities of interstellar features in several stars, taken from Table 1, together with values from the literature $(v($ lit $))$. The LWP and LWR velocities of Table 1 , being consistent with each other, have been averaged together into $v(\mathrm{LW})$.

The literature value for $\mathrm{RR}$ Tel is from Tackeray (1977). The value quoted for $\zeta$ Oph measured in the optical Ca II K and Na I D lines has been taken from Barlow et al. (1995). This value is in good agreement with the velocities derived from GHRS ultraviolet spectra by Savage et al. (1992), $-14.9 \mathrm{~km} \mathrm{~s}^{-1}$, and Brandt et al. (1996), $-15.4 \mathrm{~km} \mathrm{~s}^{-1}$. The velocities for $\eta \mathrm{UMa}$ and $\zeta$ Cas correspond to measurements of the Ca II K and Na I D optical lines reported by Vallerga et al. (1993). The velocity quoted for $\lambda$ Lep refers to the optical Ca II doublet (Frisch et al. 1990), which presents two components at 2 and $18 \mathrm{~km} \mathrm{~s}^{-1}$, which cannot be resolved with IUE. The spectrum of HD 93521 presents up to nine interstellar components, with the two strongest ones located at, approximately, -10 and $-60 \mathrm{~km} \mathrm{~s}^{-1}$ (Spitzer \& Fitzpatrick 1993). In the IUE spectra all these systems appear blended, and therefore, as in the case of $\lambda$ Lep, we cannot compare reliably the IUE velocities with the optical values.

According to the data in Table 8, the mean difference between long and short wavelength (large aperture) velocities is $17.7 \mathrm{~km} \mathrm{~s}^{-1}$, i.e. SWP velocities are systematically more negative. The mean difference between the long wavelength and the literature values is $8 \mathrm{~km} \mathrm{~s}^{-1}$.

A similar test was made to check the consistency between the wavelength scale of spectra taken through the large and small apertures. Being the number of small aperture high resolution spectra very limited, useful data were available only for the star $\zeta$ Oph in the SWP and LWR cameras. The wavelength scales of the small and large aperture spectra are fully consistent in the short wavelength range: $v(\mathrm{LAP})-v(\mathrm{SAP})=1.1 \pm 6.7 \mathrm{~km} \mathrm{~s}^{-1}$, while for the LWR camera a significant difference is found: $v(\mathrm{LAP})-v(\mathrm{SAP})=-13.7 \pm 4.1 \mathrm{~km} \mathrm{~s}^{-1}$. The lack of a suitable data set precludes an accurate determination of the offset between the large and small aperture scales in LWP spectra, but the limited tests performed seem to indicate that small aperture velocities are systematically lower, although the actual difference cannot be quantified.

The reason for the discrepancy between the short and long wavelength range velocity scales is not clear, while the large/small aperture discrepancy in LW spectra is most likely related to the transfer of the dispersion constants from the small to the large aperture: the dispersion relations were derived from spectra taken through the small aperture and then transferred to the large aperture on the basis of the assumed aperture separations.

In order to provide an internally consistent wavelength scale within the INES system, a velocity correction of $+17.7 \mathrm{~km} \mathrm{~s}^{-1}$ has been applied to the wavelength scale of SWP high resolution spectra. The wavelength scale of LWP/LWR small aperture spectra has been corrected by 
Table 8. Mean radial velocities of interstellar lines obtained from NEWSIPS SWP and LWP/LWR large aperture spectra

\begin{tabular}{lccccc}
\hline Target & $\begin{array}{c}v(\mathrm{SWP}) \\
\mathrm{km} \mathrm{s}^{-1}\end{array}$ & $\begin{array}{c}v(\mathrm{LW}) \\
\mathrm{km} \mathrm{s}^{-1}\end{array}$ & $\begin{array}{c}v \text { (lit) } \\
\mathrm{km} \mathrm{s}^{-1}\end{array}$ & $\begin{array}{c}v(\mathrm{LW})-v(\mathrm{SWP}) \\
\mathrm{km} \mathrm{s}^{-1}\end{array}$ & $\begin{array}{c}v \text { (lit) }-v(\mathrm{LW}) \\
\mathrm{km} \mathrm{s}^{-1}\end{array}$ \\
\hline RR Tel & -69.5 & -50.0 & -61.8 & 19.5 & -11.8 \\
$\zeta$ Oph & -24.7 & -11.0 & -14.8 & 13.7 & -3.8 \\
BD+28 4211 & -22.8 & -3.8 & & 19.0 & \\
BD+75 325 & -16.4 & 6.3 & & 22.7 & \\
HD 60753 & 18.8 & 31.7 & & 12.9 & \\
HD 93521 & -38.8 & -20.1 & $-10,-60$ & 18.8 & -5.2 \\
$\eta$ UMa & & 2.4 & -2.8 & & -11.5 \\
$\lambda$ Lep & & 20.1 & 2,18 & & \\
$\zeta$ Cas & & 1.2 & -10.3 & & \\
\hline
\end{tabular}

Mean difference $v(\mathrm{LW})-v(\mathrm{SWP})=17.7 \pm 3.7 \mathrm{~km} \mathrm{~s}^{-1}$.

Mean difference $v($ lit $)-v(\mathrm{LW})=-8.0 \pm 4.2 \mathrm{~km} \mathrm{~s}^{-1}$.

$+13.7 \mathrm{~km} \mathrm{~s}^{-1}$. With these corrections, the INES velocity scale is consistent with the optical determinations.

\section{Conclusions}

In this paper we have discussed the overall quality of $I U E$ high resolution spectra processed with the NEWSIPS system.

The stability of the wavelength scale $\left(\approx 5 \mathrm{~km} \mathrm{~s}^{-1}\right)$ is within the limits imposed by the acquisition and tracking accuracy. No appreciable distortions in the wavelength scale over the full spectral range or during the spacecraft lifetime have been found.

A discrepancy of $9 \mathrm{~km} \mathrm{~s}^{-1}$ has been found in the velocities derived from the two components of the Mg II doublet at $2800 \AA$ in the LWP camera. The correct velocity is provided by the $\mathrm{K}$ line measured on spectral order 83. No such discrepancy has been found in LWR spectra.

The wavelength scales of NEWSIPS short and long cameras present an inconsistency, which is well above the repeatability errors quoted above. Measurements of narrow interstellar lines have shown that SWP velocities are systematically more negative by $-17.7 \mathrm{~km} \mathrm{~s}^{-1}$, on average. A similar discrepancy has been detected in long wavelength small aperture spectra, whose velocities are more negative than those from long wavelength large aperture spectra by $-13.7 \mathrm{~km} \mathrm{~s}^{-1}$.

The determination of the inter-order background has greatly improved with respect to the the IUESIPS system, especially at the shortest wavelengths, as shown by the absence of negative fluxes in the cores of saturated absorption lines and by the greater accuracy of equivalent width measurements.

The INES system derives two spectra from each original NEWSIPS MXHI file. In the first one, the "concatenated" spectrum, the spectral orders are merged together and the overlap regions are suppressed according to an algorithm which computes suitable cut wavelengths which maximize the signal-to-noise ratio. This spectrum contains an error vector calibrated into absolute flux units, which is not available in NEWSIPS data. Since the INES high resolution spectra are obtained from NEWSIPS MXHI files, all previous considerations about the stability of the wavelength scale, the stability and accuracy of the flux scale and the validity of the background extraction are applicable to them. The second output product is the "rebinned" spectrum, which is the "concatenated" spectrum after resampling at the low resolution wavelength step.

To correct for the discrepancies found in the NEWSIPS high resolution wavelength scale, a velocity correction of $+17.7 \mathrm{~km} \mathrm{~s}^{-1}$ for SWP spectra and of $+13.5 \mathrm{~km} \mathrm{~s}^{-1}$ for LWP/LWR small aperture spectra has been applied to the INES "concatenated" spectra. With this correction, the overall INES velocity scale is self-consistent, and agrees to within $8 \mathrm{~km} \mathrm{~s}^{-1}$ with the optical velocity scale.

Acknowledgements. We would like to acknowledge the contribution of all VILSPA staff to the development and production of the INES system, and the referee, Dr. J.S. Nichols, for her useful comments.

\section{References}

Barlow M.J., Crawford I.A., Diego F., et al., 1995, MNRAS 272,333

Brandt J.C., Heap S.R., Beaver E.A., et al., 1996, AJ 112, 1128

Cassatella A., Altamore A., González-Riestra R., Ponz J.D., Barbero J., Wamsteker W., 1999, A\&AS (in press) (Paper II)

Frisch P.C., Semback K., York D.G., 1990, ApJ 364, 540

Garhart M.P., Smith M.A., Levay K.L., Thompson R.W., 1997, IUE NEWSIPS Information Manual, Version 2.0

Morton D.C., 1975, ApJ 197, 85

Morton D.C., 1991, ApJS 77, 119

Nichols J.S., 1998, in: "Ultraviolet Astronomy beyond the IUE Final Archive", ESA SP-413, Wamsteker W. and GonzálezRiestra R. (eds.), p. 671 
Nichols-Bohlin J.S., Fesen R.A., 1986, AJ 92, 642

Nichols-Bohlin J.S., Fesen R.A., 1990, ApJ 353, 281

Nichols J.S., Linsky J.L., 1996, AJ 111, 517

Rodríguez-Pascual P.M., González-Riestra R., Schartel N., Wamsteker W., 1999, A\&AS 139, 183 (Paper I) Savage B.D., Cardelli J.A., Sofia U.J., 1992, ApJ 401, 706 Smith M.A., 1999, PASP 760, 722
Spitzer L., Fitzpatrick E.L., 1993, ApJ 409, 299

Tackeray A.D., 1977, MNRAS 83, 1

Vallerga J.V., Vedder P.W., Craig N., Welsh B.Y., 1993, ApJ 411,729

Wamsteker W., Skillen I., Ponz J.D., de la Fuente A., Barylak M., Yurrita I., 1999, Ap\&SS (in press)

Zuccolo R., Selvelli P., Hack M., 1997, A\&AS 124, 425 